\title{
A SITUAÇÃO DO TRANSPLANTE DE CÉLULAS TRONCO HEMATOPOIÉTICAS NO ESTADO DO PARÁ, AMAZÔNIA, NO PERÍODO DE JANEIRO DE 2010 A DEZEMBRO DE 2015
}

\author{
The situation of transplantation of hematopoietic trunk cells in the state of Pará, \\ Amazônia in the period from January 2010 to December 2015
}

Felipe Lima Alcolumbre Tobelem¹, Maria Isabel Muniz Zemero², Lacy Cardoso de Brito Junior', Ana Cristina Simões Beltrão ${ }^{3}$, Kennie Kelly dos Santos Amazonas ${ }^{4}$

\begin{abstract}
RESUMO
Introdução: Mesmo com a expansão de unidades e leitos de transplantes de células tronco hematopoiéticas (TCTH) e laboratórios de HLA, em todo o Brasil, ainda estamos muito aquém da demanda existente. Objetivo: Entender o fluxo e caracterizar os pacientes do estado do Pará registrados no REREME, à espera de um TCTH, no período de janeiro de 2010 a dezembro de 2015. Material e Métodos: Estudo descritivo, retrospectivo, sobre o fluxo, gênero, idade e doença de base (onco-hematológica) de pacientes acima de 18 anos, cadastrados no REREME pelo estado do Pará, à espera, ou que já realizaram TCTH, a partir dos dados obtidos do REREME, da CNCDO/SESPA e Gerência de Imunogenética (GERIM) da Fundação HEMOPA. Resultados: Foi observado que 30,1\% dos pacientes estavam cadastrados no REREME (82/272); 69,8\% (190/272) realizaram pesquisa para HLA, mas não foram registrados no REREME; 54,9\% (45/82) apresentavam leucemia linfóide aguda ou mielóide aguda ou linfoma não-Hodgkin; 45,12\% (37/82) tinham entre 18-30 anos; 48,78\% (40/82) apresentavam sorologia para citomegalovirus positivo; $52,43 \%$ (43/82) eram homens; e 2,43\% (2/82) dos pacientes cadastrados realizaram TCTH fora do estado do Pará. Conclusão. Os dados obtidos mostram que, apenas com os sistemas de controle do Ministério da Saúde e REREME, não é possível o planejamento seguro de políticas públicas que embasem a necessidade de TCTH para pacientes do estado do Pará.
\end{abstract}

Descritores: Transplante de Células-Tronco Hematopoéticas; Listas de Espera; Epidemiologia.

Instituição:

1 Universidade Federal do Pará (UFPA), Belém/PA

2 Universidade do Estado do Pará (UEPA), Belém/PA

${ }^{3}$ Central Estadual de Transplantes do Pará, Belém/PA

${ }^{4}$ Fundação Centro de Hematologia e Hemoterapia do Pará Belém/PA

\section{Correspondência:}

Prof. Dr. Lacy Cardoso de Brito Junior.

Universidade Federal do Pará - Instituto de Ciências Biológicas

Lab. de Patologia Geral - Imunopatologia e Citologia.

Av. Augusto Corrêa, 01 - CEP 66075-900, Belém/PA

Fone: (91) 3201-7102

E-mail: Icdbrito@ufpa.br ou Icdbrito@bol.com.br

Recebido em: 18/09/2018

Aceito em: 19/10/2018

\section{INTRODUÇÃO}

O transplante de células tronco-hematopoiéticas I transplante de medula óssea (TCTH/TMO) é, em muitos casos, o único tratamento efetivo para algumas doenças oncohematológicas. Porém, inúmeros fatores são determinantes para o sucesso dessa terapêutica, dos quais se destacam a fase da doença em que o paciente se encontra para o transplante, o tempo entre o diagnóstico e o TCTH, o tratamento prévio adotado, o tipo de TCTH a ser realizado, tipo de profilaxia e a ocorrência da doença do enxerto contra hospedeiro (GVHD)., ${ }^{1,2}$ Mas, sem dúvida, o tempo de espera entre a busca de um doador e a realização do TCTH é um dos fatores mais determinantes da angústia do paciente..$^{3,4}$ 
Essa angústia mundial tem sido reduzida, pelo menos em parte, graças à ampliação dos Registros Internacionais de doadores não aparentados; contudo, essa esperança para os brasileiros também acaba, muitas vezes, quando se observa que a maioria dos doadores voluntários desses sistemas são caucasianos, enquanto que a população brasileira é principalmente miscigenada. ${ }^{4,8}$

Assim, para populações miscigenadas, a melhor estratégia é o fortalecimento do Registro Brasileiro de Doadores de Medula Óssea (REDOME) e da Rede de Bancos de Sangue de Cordão Umbilical (BRASILCORD), fundamentais para a obtenção de um doador compatível, nesse caso. ${ }^{3,9}$

Segundo dados históricos do Ministério da Saúde brasileiro, publicados em 2017, foram realizados pelo Sistema Único de Saúde (SUS) brasileiro, entre os anos de 2002 e 2016, 23.547 transplantes com células tronco-hematopoiéticas.

No estado do Pará, embora exista um serviço público de TCTH recentemente credenciado e autorizado (2018), e um serviço privado de TCTH que não renovou a autorização, para o mesmo período, apenas dois TCTH autólogo foram realizados, no ano de 2015, mostrando que os pacientes do estado do Pará, que precisam de TCTH, estão sendo encaminhados para outras unidades da federação. ${ }^{10}$

Assim, o objetivo deste estudo foi entender o fluxo e caracterizar os pacientes do estado do Pará registrados no Registro Brasileiro de Receptores de Medula Óssea (REREME) em espera de um TCTH, no período de janeiro de 2010 a dezembro de 2015.

\section{MATERIAL E MÉTODOS}

\section{Casuística do Estudo}

Estudo descritivo, retrospectivo, sobre o atual cenário do fluxo de pacientes cadastrados no sistema REREME pelo estado do Pará, que estão na fila de espera para um TCTH, ou que já realizaram um TCTH, a partir dos dados obtidos do próprio sistema REREME; da Central de Notificação, Captação e Distribuição de Órgãos e Tecidos (CNCDO) da Secretaria de Saúde do estado do Pará (SESPA); da Gerência de Imunogenética (GERIM), Coordenação de Ambulatórios (COAMB) e Gerência de Captação de Doadores (GECAD) da Fundação HEMOPA, no período de janeiro de 2010 a dezembro de 2015.

Os dados epidemiológicos obtidos foram: gênero, idade, doença de base (oncohematológica), presença ou não de sorologia positiva para citomegalovírus e tamanho da fila de espera para um TCTH, para pacientes maiores de 18 anos procedentes do estado do Pará nos últimos cinco anos.

\section{Análise de Dados}

Determinação de frequência de dados, média, desvio padrão, máximos e mínimos.

\section{RESULTADOS}

Na Figura 1 está o organograma que define o caminho a ser percorrido por um paciente que precisa de TCTH no estado do Pará. Neste estudo, observou-se que, após o diagnóstico de uma doença oncohematológica, e conforme indicação médica, $227 / 272(83,5 \%)$ dos pacientes foram inseridos no SUS e submetidos à pesquisa de Antígeno Leucocitário Humano (HLA), no serviço público do estado do Pará (GERIM/HEMOPA).

Figura 1. Fluxo de pacientes portadores de doenças oncohematológicas, que realizaram pesquisa de HLA, e que foram cadastrados no REREME no estado do Pará, no período de janeiro de 2010 a dezembro de 2015.

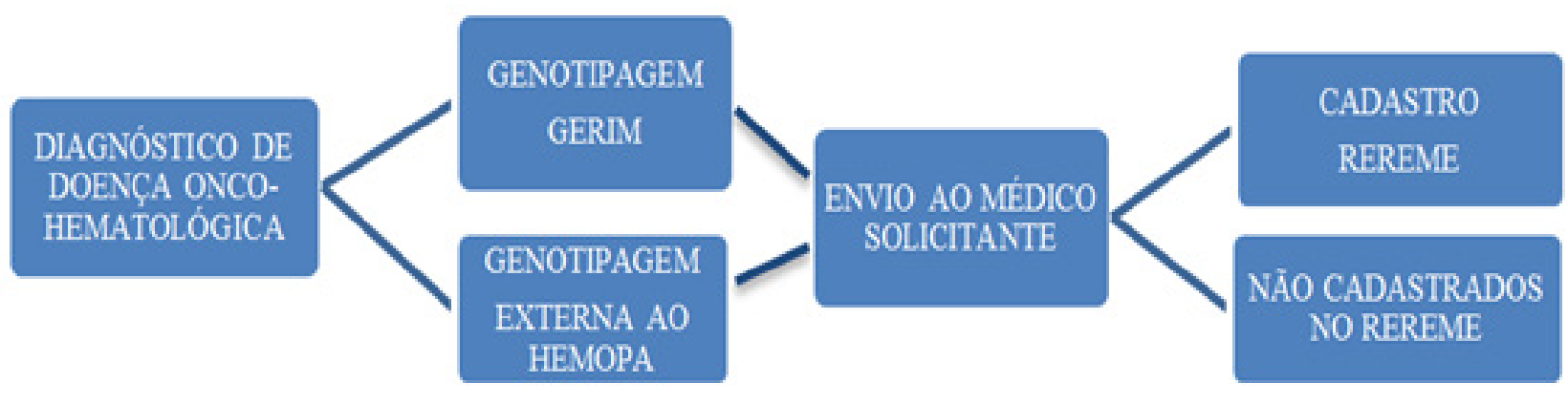

Quanto ao cadastro de pacientes no REREME, observou-se que $82 / 272(30,1 \%)$ dos pacientes foram cadastrados, porém, apenas 37/82 (45,2\%) realizaram pesquisa de HLA na GERIM/HEMOPA, conforme decisão médica. Os outros 45/82 (54,9\%) pacientes realizaram a pesquisa de HLA em outro serviço que não o público (GERIM) e, também, foram registrados no REREME e, ainda, 190/272 (69,8\%) pacientes realizaram pesquisa de HLA na GERIM/HEMOPA, porém, não foram registrados no REREME. 
Felipe Lima Alcolumbre Tobelem, Maria Isabel Muniz Zemero, Lacy Cardoso de Brito Junior, Ana Cristina Simões Beltrão, Kennie Kelly dos Santos Amazonas

Segundo dados da CNCDO/SESPA, apenas 02/82 $(2,43 \%)$ pacientes cadastrados no sistema REREME foram submetidos a TCTH no período analisado. Desses, por falta de ferramentas de registro e acompanhamento de pacientes no REREME, não foi possível avaliarem questões como a sobrevida ou mortalidade e o tipo de transplante realizado, dos pacientes transplantados para o período de análise.

Quanto ao gênero e idade dos pacientes apenas cadastrados no REREME, $39 / 82 \quad(47,56 \%)$ eram mulheres e $43 / 82(52,44 \%)$ homens, e $45,12 \%$ (37/82) encontravam-se com idade entre 18-30 anos (Tabela 1).

Tabela 1. Pacientes portadores de doenças oncohematológicas cadastrados no REREME, segundo gênero e idade, do estado do Pará, no período de janeiro de 2010 a dezembro de 2015.

\begin{tabular}{lcc}
\hline Faixa etária & Mulheres & Homens \\
\hline $18-30$ anos & 17 & 20 \\
$31-42$ anos & 12 & 8 \\
$43-54$ anos & 7 & 7 \\
$55-66$ anos & 3 & 8 \\
\hline Total & $\mathbf{3 9}(\mathbf{4 7 , 5 6 \% )}$ & $\mathbf{4 3}$ (52,44\%) \\
\hline
\end{tabular}

Fonte: REREME, CNCDO/SESPA e Gerência de Imunogenética (GERIM) da Fundação HEMOPA.

As principais doenças oncohematológicas que geraram o registro de pacientes no sistema REREME, por sua vez, estão apresentadas na Tabela 2. Destas, a leucemia linfoide aguda e a leucemia mielóide aguda, com $42 / 82(51,21 \%)$ foram as de maior prevalência em ambos os gêneros.

Tabela 2. Pacientes portadores de doenças oncohematológicas cadastrados no REREME, segundo gênero e doença de base, do estado do Pará, no período de janeiro de 2010 a dezembro de 2015.

\begin{tabular}{lcc}
\hline Doença de base & Mulheres & Homens \\
\hline Anemia Aplástica & 6 & 5 \\
Doença de Hodgkin & 6 & 2 \\
Doença Mieloproliferativa Crônica & 3 & 8 \\
Leucemia Linfoblástica Aguda & 11 & 7 \\
Leucemia Mielóide Aguda & 10 & 14 \\
Linfoma não-Hodgkin & 0 & 3 \\
Micose Fungóide & 1 & 0 \\
Síndrome Mielodisplasica & 2 & 4 \\
\hline TOTAL & $\mathbf{3 9}$ (47,56\%) & $\mathbf{4 3}$ (52,44\%) \\
\hline
\end{tabular}

Fonte: REREME, CNCDO/SESPA e Gerência de Imunogenética (GERIM) da Fundação HEMOPA.
Essa análise também levou em consideração o número de pacientes cadastrados no REREME que apresentavam sorologia para citomegalovírus (CMV). $\mathrm{Na}$ Tabela 3, observa-se que 40/82 (48,78\%) apresentavam sorologia positiva para CMV, dos quais, 24/40 (60\%) eram indivíduos do gênero masculino, distribuído em várias faixas etárias.

Tabela 3. Pacientes portadores de doenças oncohematológicas cadastrados no REREME, segundo a presença de sorologia para citomegalovírus em relação ao gênero e a faixa etária, do estado do Pará, no período de janeiro de 2010 a dezembro de 2015.

\begin{tabular}{lcccccc}
\hline Faixa Etária & \multicolumn{3}{c}{ Mulheres } & \multicolumn{3}{c}{ Homens } \\
\hline & $\mathrm{S} / \mathrm{S}$ & $\mathrm{CMV}+$ & $\mathrm{CMV}-$ & $\mathrm{S} / \mathrm{S}$ & $\mathrm{CMV}+$ & $\mathrm{CMV}-$ \\
\hline 18-30 anos & 1 & 9 & 7 & 4 & 8 & 8 \\
31-42 anos & 2 & 4 & 6 & 1 & 7 & 0 \\
$43-54$ anos & 2 & 2 & 3 & 0 & 5 & 2 \\
$55-66$ anos & 0 & 1 & 24 & 1 & 4 & 3 \\
\hline Total & $\mathbf{5}$ & $\mathbf{1 6}$ & $\mathbf{1 8}$ & $\mathbf{6}$ & $\mathbf{2 4}$ & $\mathbf{1 3}$ \\
\hline
\end{tabular}

Legenda. *S/S-Sem dados de Sorologia para Citomegalovírus registrados; $\mathrm{CMV+}$ sorologia positiva para citomegalovírus; CMV-sorologia negativa para citomegalovírus.

Fonte: REREME, CNCDO/SESPA e Gerência de Imunogenética (GERIM) da Fundação HEMOPA.

\section{DISCUSSÃO}

A análise do organograma que define o caminho a ser percorrido por um paciente do estado do Pará, antes do TCTH, mostrou que a forma de acesso dos pacientes ao REREME não difere do que ocorre em outros estados do Brasil. ${ }^{3,9}$

Quanto à análise de dados do REREME, apenas dois pacientes dos 82 cadastrados foram transplantados. Os transplantes foram realizados no estado de São Paulo, na Casa de Saúde Santa Marcelina e no Hospital Israelita Albert Einstein. Em relação ao perfil dos indivíduos transplantados, observou-se um paciente do sexo feminino, 43 anos de idade, portador de Leucemia Mielóide Crônica (Cid C92.1) e outro do sexo masculino, 65 anos de idade, portador de Síndrome Mielodisplásica, não especificada (Cid D46.9).

Não foram obtidas informações quanto ao tipo de transplante realizado e índice de sobrevida ou óbito de pacientes, em função da falta de ferramentas e informações no sistema ou em qualquer outra unidade controladora, conforme pode ser constatado nos boletins do Registro Brasileiro de Transplantes, da Associação Brasileira de Transplante de Órgãos ${ }^{11-17}$ e dados do Ministério da Saúde/BR. ${ }^{10}$ 
Os dados obtidos dos arquivos da CNCDO/SESPA para o período revelaram que o total de pacientes transplantados do estado do Pará foi de 02/82 casos, o que condiz com os registros do Ministério da Saúde brasileiro para o mesmo período. ${ }^{10}$

Contudo, há uma contradição de dados quando são analisados os boletins do Registro Brasileiro de Transplantes da Associação Brasileira de Transplante de Órgãos para os anos de 2005 até março de 2018, mesmo tendo como fonte geradora das informações locais a CNCDO/SESPA. Segundo esses boletins, nenhum TCTH foi realizado com pacientes do estado do Pará no período analisado. ${ }^{11-17}$

Beltrão, Tavares, Soares (2006), ${ }^{18}$ por sua vez, em seus estudos sobre o TCTH com pacientes provenientes da região metropolitana de Belém - Pará, no período de 2001 a 2005, observaram que a média de idade destes foi de 27,6 anos; a aplasia medular foi a doença de base mais frequente e ainda que a maioria dos TCTH foi realizada no estado de São Paulo, tendo sido a doença do enxerto contra o hospedeiro (DECH) a principal complicação observada. Esses autores relatam, porém, que os dados foram obtidos junto à Fundação HEMOPA e ao Hospital Ophir Loyola, locais de acompanhamento dos pacientes pós-TCTH. Contudo, embora em nossos estudos também tenhamos obtido dados junto à Fundação HEMOPA, esses dados estavam muito incompletos e, algumas vezes, restritos aos prontuários médicos do paciente, o que restringiu acesso a informações do pósoperatório desses pacientes.

Também em nossos estudos, observamos que a maioria dos pacientes apresentavam-se com idade entre 18-30 anos, porém, principalmente associados a casos de leucemias agudas mielóides e linfóides, e sem diferença em relação aos gêneros, semelhante ao que tem sido descrito na literatura. . $^{2,11-17,19-21}$

Fato relevante neste estudo é o numero significativo de pacientes $(48,78 \%)$ que apresentavam sorologia positiva para CMV. Este dado deve ser sempre analisado com atenção, visto que pacientes indicados a transplantes com infecções ativas para CMV têm risco aumentado de desenvolver neutropenia persistente e ser acometido por infecções bacterianas. ${ }^{22,23}$

\section{CONCLUSÃO}

Os dados obtidos mostram que, nem no passado e nem hoje, é possível o planejamento seguro de políticas públicas que embasem a necessidade de TCTH para pacientes do estado do Pará, visto não ser possível, apenas com o sistema de controle do Ministério da Saúde e Registro Brasileiro de Receptores de Medula Óssea (REREME), definir o tamanho real da fila de espera de pacientes para o TCTH e nem a atual capacidade do estado em atender essa demanda.

\section{ABSTRACT}

Introduction: Even with the expansion of units and beds for hematopoietic stem cell transplants (HSCT), and HLA laboratory, throughout Brazil, we are still far short in relation to the existing demand. Purpose: To understand the flow and characterize patients from the State of Pará registered in the REREME waiting for a HSCT from January 2010 to December 2015. Material and Methods: A descriptive, retrospective study on the flow gender, age and underlying disease (oncohematologic) of patients above 18 years of age, enrolled in the REREME by the State of Pará, waiting, or who have already performed HSCT from data obtained from REREME, CNCDO / SESPA and Immunogenetics Management (GERIM) of the HEMOPA Foundation. Results: It was observed that $30.1 \%$ patients were enrolled in REREME (82/272); 69.8\% (190/272) performed research for HLA, but were not enrolled in REREME; 54.9\% (45/82) presented acute lymphoid leukemia or acute myeloid leukemia or non-Hodgkin's lymphoma; $45.12 \%(37 / 82)$ were between 18-30 years old; $48.78 \%$ (40/82) presented positive cytomegalovirus sorology; $52.43 \%$ (43/82) were men; and $2.43 \%$ (2/82) of the HSCT were performed outside the State of Pará. Conclusion: Data obtained show that this is not possible just using control systems of the Ministry of Health and REREME, the safe planning of public policies to support the need for HSCT for patients from the state of Pará.

Keywords: Hematopoietic Stem Cell Transplantation; Waiting List; Epidemiology. 
Felipe Lima Alcolumbre Tobelem, Maria Isabel Muniz Zemero, Lacy Cardoso de Brito Junior, Ana Cristina Simões Beltrão, Kennie Kelly dos Santos Amazonas

\section{REFERÊNCIAS}

1. Seber A, Castro HC, Borsato ML, Matsuo SE, Pires AL, Junior CGC, et al. Transplante de células-tronco hematopoiéticas em pediatria no Brasil. Rev. Bras. Hematol. Hemoter. 2008;30(supl 3):p.70-1.

2. Seber A. O transplante de células-tronco hematopoéticas na infância: situação atual e Perspectivas. Rev. Bras. Hematol. Hemoter. 2009;3(Supl. 1):59-67.

3. Bouzas LFS, Petersdorf EW, Anasetti C, Martin PJ, Gooley T, Radich J, Malkki M, et al. Limits of HLA mismatching in unrelated hematopoieticcell transplantation. Blood. 2004;104(9):297680;

4. Saboya R, Dulley FL, Ferreira E, Simões B. Transplante de medula óssea com doador familiar parcialmente compatível. Rev. Bras. Hematol. Hemoter. 2010;32(1):13-5.

5. Tabak D. Transplante de medula óssea em leucemia mielóide aguda - resultados preliminares do grupo cooperativo brasileiro. Rev. Bras. Hematol. Hemoter. 2006;28(1):1-4.

6. Nicolau JE, Melo LMMP, Sturaro D. Avaliação da alta precoce após transplante alogênico de medula óssea para leucemia mielóide crônica. São Paulo Medical Journal. 2007;125(3):1749.

7. Neto AEH, Pereira J. Linfoma de Hodgkin recidivado após transplante autólogo de medula óssea. Rev. Bras. Hematol. Hemoter. 2008; 30(4):259-265.

8. Tiercy JM, Nicoloso G, Passweg J. The probability of identifying a 10/10 HLA allele-matched unrelated donor is highly predictable. Bone Marrow Transplant. 2007;40(6):51522.

9. Bouzas LFS. Análise da capacidade do REDOME/RENACORD em suprir as necessidades dos pacientes registrados no REREME [Tese - Doutorado]. Rio de Janeiro (RJ): Instituto Nacional de Câncer - Pós-Graduação em Oncologia, 2011.

10.BRASIL. Ministério da Saúde. Sistema Nacional de Doação e Transplante de Órgãos. 2018 [Acessado em 08 de junho de 2018]. Disponível em http://portalms.saude.gov.br/acoese-programas/doacao-transplantes-deorgaos/servicos/ estatisticas

11. RBT 2012 (JAN/DEZ) - ABTO, Dimensionamento dos Transplantes no Brasil e em cada Estado (2005-2012), páginas 14 e 52. p. 1-98.
12.RBT 2013 (JAN/DEZ) - ABTO, Dimensionamento dos Transplantes no Brasil e em cada Estado (2006-2013), páginas 14 e 52. p. 1-79.

13.RBT 2014 (JAN/DEZ) - ABTO, Dimensionamento dos Transplantes no Brasil e em cada Estado (2007-2014), páginas 14 e 52. p. 1-98.

14.RBT 2015 (JAN/DEZ) - ABTO, Dimensionamento dos Transplantes no Brasil e em cada Estado (2008-2015), páginas 14 e 54. p. 1-97.

15.RBT 2016 (JAN/DEZ) - ABTO, Dimensionamento dos Transplantes no Brasil e em cada Estado (2009-2016), páginas 14 e 56. p. 1-102.

16.RBT 2017 (JAN/DEZ) - ABTO, Dimensionamento dos Transplantes no Brasil e em cada Estado (2010-2017), páginas 14 e 54. p. 1-99.

17. RBT 2018 (JAN/MARÇO) - ABTO, Dimensionamento dos Transplantes no Brasil e em cada Estado, páginas 11. p. 1-20.

18. Beltrão ACS, Tavares GF, Soares JS. Clínica e epidemiologia de pacientes submetidos ao transplante de medula óssea. Revista Paraense de Medicina. 2006;20(3):27-31.

19. Bouzas LFS. Transplante de medula óssea em pediatria e transplante de cordão umbilical. Ribeirão Preto: Medicina. 2000; 33:241-63.

20. Mastropietro AP. Impacto da DECH crônica na qualidade de vida pós-TCTH alogênico. Rev Bras Hematol Hemoter. 2010;32(5):358-64.

21. Abreu MHNG, Oliveira IR, Resende RG, Cardoso NMM, Correia-Silva JF, Gomez RS. - Análise Sociodemográfica e Clínica de Pacientes Submetidos ao Transplante Alogênico de Células-Troncos. Pesq Bras Odontoped Clin Integr, João Pessoa. 2012;12(3):345-50.

22.Saboya R. Infecções bacterianas e fúngicas no transplante de medula óssea - análise de 186 pacientes [Tese]. São Paulo (SP): Universidade de São Paulo - Faculdade de Medicina, 1998.

23. Benjamin DK Jr, Miller WC, Bayliff S, Martel L, Alexander $\mathrm{KA}$, Martin PL. Infections diagnosed in the first year after pediatric stem cell transplantation. Pediatr Infect Dis J. 2002;21:227-34 